\title{
Hiperglicemia materna diária diagnosticada pelo perfil glicêmico: um problema de saúde pública materno e perinatal
}

\author{
Maternal daily hyperglycemia diagnosed by glycemic profile: a maternal and \\ perinatal public health problem
}

\begin{abstract}
Marilza Vieira Cunha Rudge ${ }^{1}$, Iracema de Mattos Paranhos Calderon ${ }^{1}$, Maria Delgi Ramos ${ }^{1}$, Maria Aparecida Mourão Brasil ${ }^{1}$, Lígia Maria Suppo Souza Rugolo ${ }^{2}$, Grasiela Bossolan ${ }^{2}$, Jon Øyvind Odland ${ }^{3}$
\end{abstract}

\section{RESUMO}

Este trabalho constitui uma síntese e revisão dos principais resultados das pesquisas, com o objetivo de validar o grupo IB de Rudge. As gestantes deste grupo têm rastreamento positivo e diagnóstico negativo para o diabete gestacional, ou seja, apresentam resposta normal ao teste oral de tolerância à glicose (TTG100g). Apesar disso, as alterações nas glicemias ao longo do dia, confirmadas no perfil glicêmico (PG) caracterizam a hiperglicemia diária, fator responsável por risco materno e desfecho perinatal adverso. Estas gestantes são erroneamente incluídas na categoria de "pré-natal de baixo risco" e não estão sendo diagnosticadas e tratadas. Correspondem a 13,8\% da população de gestantes rastreadas que, somados aos 7,0\% das gestações complicadas por diabete, aumentam a ocorrência de distúrbios hiperglicêmicos na gestação para cerca de $20,0 \%$. Tem índice de mortalidade perinatal de 41\%o, semelhante ao de gestantes diabéticas e 10 vezes maior que o de não diabéticas; suas placentas apresentam alterações morfológicas e funcionais diferenciadas dos grupos de gestantes não diabéticas e diabéticas, indicativas de ajuste para manutenção da atividade funcional, facilitando a passagem de glicose para o feto e explicando a macrossomia fetal (53,8\% das gestantes não tratadas). O risco materno para hipertensão, obesidade e hiperglicemia é elevado e parece reproduzir modelo da síndrome metabólica, favorecendo risco potencial para diabete no futuro; 10 anos após a gravidez-índice, o diabete clínico tipo 2 foi confirmado em 16,7\% das mulheres do grupo IB. Os autores propõem o desenvolvimento de projetos multicêntricos, para identificar biomarcadores, de múltiplos enfoques, específicos das gestantes do grupo IB de Rudge e estabelecer protocolos para o diagnóstico dos distúrbios hiperglicêmicos da gestação, padronizando a associação TTG100g + PG, conduta que poderá causar impacto na morbimortalidade perinatal das gestações complicadas por hiperglicemia diária.

PALAVRAS-CHAVE: Gravidez; Diabetes melito; Hiperglicemia; Prognóstico; Saúde pública

\section{ABSTRACT}

This is both a synthesis and a review of the major research findings, with the aim of validating Rudge's group IB. In this group of pregnants, screening for gestational diabetes was positive while the diagnosis was negative (normal 100 g-oral glucose tolerance test 100 g-OGTT). Nonetheless, the variations in glucose levels observed throughout the day, and confirmed by the glycemic profile (GP), characterized diurnal hyperglycemia, which accounts for maternal risk and adverse perinatal outcome. The description of this group is unique for both the establishment of the diagnosis during gestation and the follow-up of both the mother and the infant. These pregnancies have been erroneously classified as "low risk" and have not been diagnosed or treated. The IB group corresponds to $13.8 \%$ of the pregnant women screened in our service. This rate, added to the $7 \%$ of pregnancies complicated by diabetes, increase the occurrence of hyperglycemic disorders during gestation to up to $20.0 \%$. In Rudge's group IB: a) perinatal mortality rate is $41 \%$, which is similar to that observed among diabetic pregnant women and 10 times higher than that found among non-diabetics; b) the observed placental abnormalities (both morphological and functional) differed from those seen in nondiabetic and diabetic pregnant women, indicating an adjustment to maintain functional activities that facilitated the passage of glucose to the fetus and explained fetal macrosomia (53.8\% in non-treated pregnancies); c) maternal risk for hypertension, obesity and hyperglycemia was high and seemed to reproduce a model of metabolic syndrome, favoring the potential risk for future

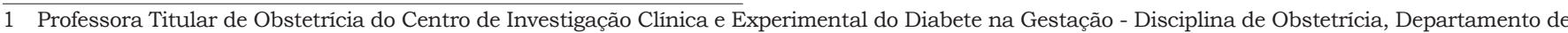
Ginecologia e Obstetrícia da Faculdade de Medicina de Botucatu - Universidade Estadual Paulista "Júlio de Mesquita Filho" - UNESP - Botucatu (SP) - Brasil.

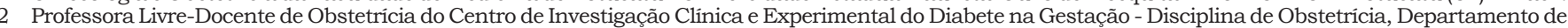
Ginecologia e Obstetrícia da Faculdade de Medicina de Botucatu - Universidade Estadual Paulista "Júlio de Mesquita Filho" - UNESP - Botucatu (SP) - Brasil.

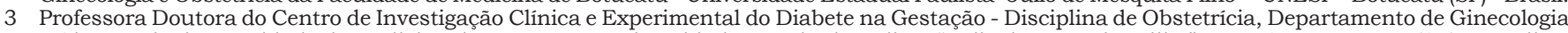
e Obstetrícia da Faculdade de Medicina de Botucatu - Universidade Estadual Paulista "Júlio de Mesquita Filho" - UNESP - Botucatu (SP) - Brasil.

4 Professora Titular da Faculdade de Ciências Agronômicas e Engenharia Florestal de Botucatu - Botucatu (SP) - Brasil.

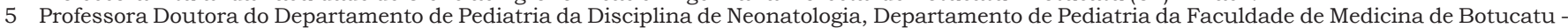
Universidade Estadual Paulista“Júlio de Mesquita Filho" - UNESP - Botucatu (SP) - Brasil.

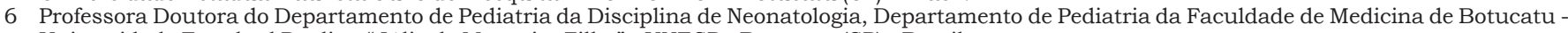
Universidade Estadual Paulista“Júlio de Mesquita Filho" - UNESP - Botucatu (SP) - Brasil.

7 Pesquisador visitante do Institute of Community Medicine, University of Tromsø / Norway.

Correspondência: Iracema de Mattos Paranhos Calderon

Rua Atílio Losi, 226 - Jd. Paraíso - 18610-260 - Botucatu - SP - e-mail: calderon@fmb.unesp.br 
diabetes; d) 10 years after the index-pregnancy, type 2 diabetes was confirmed in $16.7 \%$ of the women in group IB. The authors suggest the development of multicentric studies in order to identify biomarkers specific for Rudge's group IB and establish protocols for the diagnosis of gestational hyperglycemic disorders using the combination GP $+100 \mathrm{~g}$-GTT as a standard. This procedure may cause an impact on the morbidity/mortality rate among pregnancies complicated by diurnal hyperglycemia.

KEYWORDS: Pregnancy; Diabetes mellitus; Hyperglycemia; Prognosis; Public health

\section{Introdução}

A obstetrícia atual enfoca a diminuição do risco de morte materna e perinatal e de doença atual e futura na mãe e no recém-nascido. A moderna assistência pré-natal utiliza o rastreamento, o diagnóstico, o tratamento, e o seguimento do binômio mãe-recém-nascido para inúmeras intercorrências clínicas ${ }^{1}$. É consenso que durante a gravidez o diabete melito, uma das intercorrências clínicas, está associado a risco aumentado de resultado perinatal indesejável. Entretanto, este risco é controverso nos casos de intolerância materna à glicose de graus menos graves que o diabete melito ${ }^{2}$. Esta dúvida tem contribuído para a polêmica sobre o rastreamento e o diagnóstico do diabetes na gestação.

A Associação Americana de Diabete $(\mathrm{ADA})^{3}$ preconiza a realização do teste oral de sobrecarga com $50 \mathrm{~g}$ de glicose (TTG50g), entre a $24^{\mathrm{a}}$ e a $28^{\mathrm{a}}$ semana de gestação, avaliando a concentração de glicose plasmática uma hora após a ingestão. Este rastreamento deve ser seletivo para mulheres de risco, considerando a idade materna acima de 25 anos, a obesidade prévia ou o ganho de peso excessivo na gestação atual, história familiar de diabete (parentes de primeiro grau), história obstétrica ruim (óbito intra-útero sem causa aparente, polidrâmnio, macrossomia ou malformação fetal, pré-eclâmpsia ou eclâmpsia), história prévia de tolerância anormal à glicose e ascendência étnica de alta prevalência de diabete. O Grupo de Trabalho do Diabetes Gestacional (GTDG) do Brasil, reconhecendo a facilidade do rastreamento pela glicemia de jejum associada a fatores de risco, sugere a avaliação da glicemia de jejum a partir da $20^{a}$ semana de gestação, tendo como ponto de corte $85 \mathrm{mg} / \mathrm{dL}$. Destaca, ainda, a importância deste exame na primeira consulta de pré-natal, especialmente nas portadoras de fatores de risco para o diabete melito ${ }^{4}$.

A associação da glicemia de jejum a fatores de risco (antecedentes pessoais, familiares e obstétricos investigados na primeira consulta) foi proposta há mais de $20 \operatorname{anos}^{5}$. Se a glicemia de jejum for inferior a $90 \mathrm{mg} / \mathrm{dL}$ e a paciente não tiver qualquer antecedente, ela é considerada normal do ponto de vista de risco para o diabete melito gestacional. Se a glicemia de jejum for superior ou igual a este limite, independentemente da presença dos antecedentes, ou se a glicemia de jejum for normal, mas a paciente tem fator de risco, o rastreamento é considerado positivo e ela é encaminhada para a confirmação do diagnóstico, entre a $24^{a}$ e a $26^{a}$ semana de gestação. A comparação entre o rastreamento universal, o TTG50g, e a associação glicemia de jejum-fator de risco, método mais simples e de fácil realização, confirmou equivalência entre eles ${ }^{6}$. A associação glicemia de jejum-fator de risco tem sensibilidade de $83,7 \%$ e valor preditivo negativo de $95,3 \%$ em relação ao TTG50g7.

Para a confirmação do diagnóstico, a $\mathrm{ADA}^{3}$ recomenda o teste oral com sobrecarga de $100 \mathrm{~g}$ de glicose (TTG100g) para as gestantes que excederam o valor limite de $140 \mathrm{mg} / \mathrm{dL}$ no TTG50g. Define como limites de normalidade para este exame 95, 180, 155 e $140 \mathrm{mg} / \mathrm{dL}$, respectivamente para o jejum, uma, duas e três horas após a ingestão de glicose. Dois valores iguais ou superiores confirmam o diabete gestacional. Admite a alternativa da sobrecarga de $75 \mathrm{~g}$ de glicose (TTG75g), à semelhança do diagnóstico realizado fora do periodo gestacional. Entretanto, considera para o resultado os mesmos critérios da gestação, ou seja, dois valores iguais ou superiores a 95, 180 e 155, respectivamente para o jejum, 1 e 2 horas pós-sobrecarga. A Organização Mundial de Saúde $^{8}$ preconiza a sobrecarga de 75 g (TTG75g), delimitando como pontos de corte a glicemia de jejum $(\geq 126 \mathrm{mg} / \mathrm{dL})$ e a glicemia de duas horas pós-sobrecarga ( $\geq 140 \mathrm{mg} / \mathrm{dL}$ ). Classifica as gestantes como portadoras de intolerância à glicose (glicemia de duas horas entre 140 e $200 \mathrm{mg} / \mathrm{dL}$ ) ou de diabete gestacional (glicemia de duas horas superior ou igual a $200 \mathrm{mg} / \mathrm{dL}$ ).

No Brasil, o GTDG padronizou o TTG75g, com os limites de $110 \mathrm{mg} / \mathrm{dL}$ para a glicemia de jejum e de $140 \mathrm{mg} / \mathrm{dL}$ para o valor de duas horas após sobrecarga, incluindo a categoria "glicemia de jejum alterada" na classificação do diabete na gestação ${ }^{4}$. No entanto, Rudge et al. ${ }^{9}$, utilizando dois testes diagnósticos em paralelo, identificaram quatro grupos de gestantes com respostas diferenciadas ao TTG100g associado ao perfil glicêmico 
(PG). Esse exame consiste na avaliação de glicemias plasmáticas maternas a cada duas horas, das 8 às 18 horas, com dieta padronizada de 2840 calorias, distribuídas em cinco refeições. Os valores de normalidade são de $90 \mathrm{mg} / \mathrm{dL}$ para a glicemia de jejum e $130 \mathrm{mg} / \mathrm{dL}$ para as pós-prandiais. Apenas um resultado alterado confirma a resposta inadequada ao teste, ou seja, a hiperglicemia diária. A associação do TTG100g ao PG definiu uma nova classificação diagnóstica para o diabete associado à gestação. As gestantes do grupo IA são consideradas euglicêmicas, ou seja, não diabéticas. As dos grupos IIA e IIB são diabéticas pela literatura, que preconiza o TTG100g para o diagnóstico:- as do grupo IIA são diabéticas gestacionais (Classe A) e as do grupo IIB, diabéticas gestacionais (Classes A ou A/B) ou clinicas (Classes B a FRHT). Apesar do TTG normal, as gestantes do grupo IB apresentam hiperglicemia ao longo do dia, diagnosticada pelo PG alterado. Não têm correspondência com a classificação de White, mas têm intolerância a carboidratos, proteínas e lipídios e devem ser tratadas com dieta e insulina para controlar a hiperglicemia diária ${ }^{10}$.

O grupo IB foi acidentalmente identificado em 1983 em estudo prospectivo para padronização do PG, comparando-o com o TTG100g no diagnóstico do diabete na gestação ${ }^{9}$. Este grupo específico foi incluído no protocolo de diagnóstico e tratamento das gestações complicadas por diabete ou hiperglicemia diária, no Serviço de Diabete e Gravidez da Disciplina de Obstetrícia da Faculdade de Medicina de Botucatu-UNESP.

O objetivo desta revisão é mostrar os resultados relativos às gestantes do grupo IB que, apesar de classificadas como baixo risco, têm resultado materno e perinatal adverso, decorrente da hiperglicemia diária diagnosticada no PG. A partir de 1996, todos os projetos citados no texto foram aprovados pelo Comitê de Ética em Pesquisa em Seres Humanos da FM de Botucatu-UNESP.

\section{Resultados Relevantes e Discussão}

\section{Caracterização do grupo IB de Rudge}

O grupo IB corresponde a gestantes portadoras de hiperglicemia diária, ou seja, as que apresentam rastreamento positivo mas sem diagnóstico de diabete gestacional (TTG100g normal), apesar de alterações no $\mathrm{PG}^{9}$.

O TTG100g é o teste diagnóstico para diabete gestacional recomendado na literatura atual ${ }^{3}$. O PG foi descrito por Gillmer et al. ${ }^{10}$, para quantificar os níveis glicêmicos maternos diários, com a ingestão de dieta de 2500 calorias. Testa a resposta glicêmica à associação de carboidratos, proteínas e lipídios, por avaliações da glicemia plasmática materna a cada duas horas, das 8 às 18 horas (Figura 1).

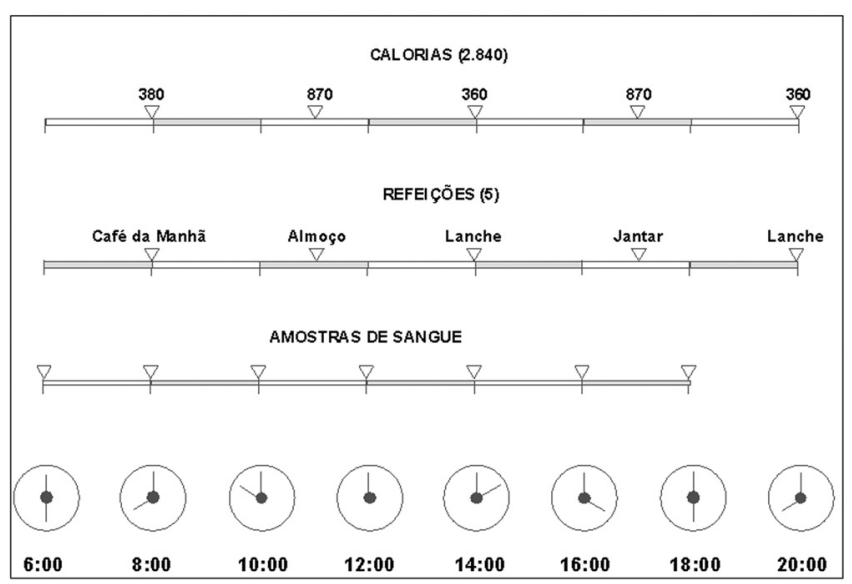

Figura 1 - Esquema para a determinação do perfil glicêmico, indicando momento e descrição da ingestão alimentar e da coleta de sangue ${ }^{9,10}$.

A análise dos resultados obtidos em 192 gestantes às quais foram aplicados o TTG $100 \mathrm{~g}$ e o PG, de forma paralela e independente, diferenciou quatro grupos de gestantes, denominados por Rudge et al. ${ }^{9}$ de Grupo IA - TTG 100 g e PG normais $(\mathrm{n}=79 ; 41,1 \%)$, grupo IB - TTG100g normal e PG alterado ( $\mathrm{n}=63 ; 32,8 \%)$, grupo IIA - TTG100g alterado e PG normal ( $\mathrm{n}=18 ; 9,4 \%)$, grupo IIB - TTG $100 \mathrm{~g}$ e PG alterados ( $n=32 ; 16,7 \%)$.

Diante da hipótese inicial, que considerava a superposição das respostas a ambos os testes, tais resultados foram surpreendentes. Mas ficou a dúvida - quem tratar? Considerando o desfecho de peso fetal como marcador da necessidade de controle da hiperglicemia e introdução de terapêutica nas gestações complicadas pelo diabete, o grupo IB de Rudge et al. ${ }^{9}$ apresentou $53,8 \%$ de recémnascidos grandes para idade gestacional (GIG) e/ ou macrossômicos. Esta proporção foi estatisticamente semelhante aos $51,9 \%$ observados no grupo IIB e maior que as dos grupos IA $(25,6 \%)$ e IIA $(28,6 \%)$.

A acurácia diagnóstica do TTG100g e do PG para o desfecho de macrossomia/GIG é mostrada na Tabela 1.

Tabela 1 - Acurácia do teste oral de tolerância à glicose (TTG100g) e do perfil glicêmico (PG) para o diagnóstico de macrossomia/grandes para a idade gestacional ${ }^{9}$.

\begin{tabular}{lcc}
\hline Parâmetros (\%) & TTG100g & PG \\
\hline Sensibilidade & 36,0 & 68,0 \\
Especificidade & 17,8 & 12,3 \\
Valor preditivo positivo & 36,0 & 68,0 \\
Valor preditivo negativo & 17,8 & 100,0 \\
\hline
\end{tabular}


A hiperglicemia materna leva à hiperglicemia e à hiperinsulinemia fetal, resultando em crescimento exagerado do feto ${ }^{11,12}$. O crescimento fetal é mediado pela hiperinsulinemia fetal, pelo excesso de glicose e nutrientes que atravessam ou são secretados pela placenta ${ }^{13}$, e potencialmente modificado por fatores genéticos ${ }^{2}$. A macrossomia é a complicação mais freqüente entre os filhos de mãe diabética, com graves repercussões perinatais e prevalência variável, diretamente relacionada aos níveis de glicemia materna ${ }^{14}$.

Os tocotraumatismos, decorrentes da desproporção de ombro e tórax, os distúrbios metabólicos e eletrolíticos neonatais, como a hipoglicemia e a hipocalcemia, e a hipóxia periparto são complicações diretamente relacionadas aos níveis maternos elevados de glicose. Esses fetos macrossômicos têm risco duas vezes maior de distocia de ombro no parto vaginal e também grande chance de morte intra-uterina nas últimas quatro semanas de gestação ${ }^{15,16}$.

As taxas de macrossomia se alteraram pouco nas últimas décadas, provavelmente pela baixa sensibilidade do TTG100g em detectar a macrossomia fetal. O estudo observacional e prospectivo Hyperglycemia and Adverse Pregancy Outcome (HAPO) $^{2}$, em desenvolvimento desde 2002, com inclusão prevista de 25000 gestantes, alerta para a interação da hiperglicemia de graus variados com o prognóstico perinatal adverso, incluindo aumento das taxas de cesárea, macrossomia fetal, hipoglicemia neonatal e hiperinsulinismo fetal. A literatura tem reforçado a idéia de que a hiperglicemia de intensidade variada, independentemente do diagnóstico de diabete gestacional, está envolvida na determinação do peso ao nascimento. Na casuística do nosso serviço, a hiperglicemia diária, identificada por alterações no PG, foi confirmada em $62,4 \%$ e o diabete gestacional, diagnosticado por alterações no TTG100g, correspondeu a apenas $15,5 \%$ do total de mães de 315 recém-nascidos macrossômicos ${ }^{17-19}$. Resultados semelhantes com a identificação de um grupo de gestantes com TTG100g normal e PG alterado foram apresentados por um grupo holandês no Congresso Mundial de Ginecologia e Obstetrícia do Rio de Janeiro e posteriormente publicados ${ }^{20}$.

\section{Prevalência e implicações clínicas do grupo IB de Rudge}

Após dez anos de implementação deste protocolo diagnóstico (1985-1994), confirmou-se que $20,4 \%$ das gestantes apresentam alterações em um ou nos dois testes diagnósticos - 13,8\% correspondem ao grupo IB (PG alterado), 0,8\% ao grupo IIA (TTG100g alterado) e 5,9\% ao grupo IIB (TTG 100g e
PG alterados) ${ }^{21}$. Estes indicadores aumentam muito a proporção de gestações complicadas por hiperglicemia materna que, independentemente do teste diagnóstico, devem ser identificadas e tratadas.

O tratamento com dieta, insulina e atividade física diminuiu a incidência de macrossomia no grupo IB, atingindo cifras de $29,5 \%$. Esta proporção é semelhante à observada no grupo IA, de gestantes com respostas normais aos dois testes diagnósticos ${ }^{22}$.

Na tentativa de esclarecer porque o TTG100g não é capaz de predizer macrossomia fetal, foi analisado o Banco de Dados do Serviço de Diabete e Gravidez da Faculdade de Medicina de BotucatuUNESP, e as gestantes foram reclassificadas de acordo com novos limites para o TTG $100 \mathrm{~g}$ e para o PG. Foram testados três pontos de corte para o TTG100g (média, média+um desvio padrão e média+dois desvios-padrão) e dois para o PG (média e média+um desvio padrão). A comparação dos intervalos de confiança da acurácia dos pontos de corte mostrou que o melhor critério para diagnosticar a macrossomia fetal é o que utiliza os valores da média para o TTG100g e da média+um desvio padrão para o $\mathrm{PG}^{22}$, ou seja, limites inferiores aos recomendados na literatura.

O coeficiente de mortalidade perinatal foi semelhante nos grupos IB, IIA e IIB, correspondentes a, respectivamente, $41 \%$, 60\% e $64 \%$ nascidos vivos, dez vezes maior que o observado no grupo IA ${ }^{15,16}$. Tais resultados reforçaram a decisão da continuidade das investigações, pois as gestantes do grupo IB, consideradas normais pela literatura, têm mortalidade perinatal comparável aos grupos diabéticos.

Em projeto conjunto com o Serviço de Neonatologia da Faculdade de Medicina de Botucatu, foram avaliados 245 recém-nascidos, filhos de mães portadoras de diabete gestacional (classes A e A/B de White; $n=129$ ) ou de hiperglicemia diária (grupo IB; $n=116$ ), submetidas ao mesmo protocolo de tratamento durante a gestação. Não se encontrou diferença significante entre os grupos quanto à ocorrência de prematuridade, macrossomia/GIG $(29,4 \%$ para o diabete gestacional e $28,4 \%$ para o grupo IB), sindrome do desconforto respiratório e hipoglicemia neonatal. As taxas de morte fetal e neonatal, e conseqüentemente, o indice de morte perinatal, foram semelhantes ${ }^{23}$. Estes resultados evidenciaram que os recém-nascidos de mães portadoras de hiperglicemia diária (grupo IB) têm risco potencial para as mesmas complicações perinatais do diabete gestacional. Além disso, confirmaram a necessidade de instituir terapêutica com dieta e insulina para o controle da hiperglicemia materna. 


\section{Repercussões placentárias da hiperglicemia materna diária}

As alterações morfológicas das placentas de pacientes do grupo IB são semelhantes às observadas nas placentas de gestantes diabéticas. Há maior incidência de endarterite; característica histopatológica considerada por Benirschke como uma lesão post-mortem ${ }^{21,22}$. Esse achado associase à maior freqüência de mortalidade perinatal, daí a necessidade de ser melhor investigado e confirmado por outros autores.

O estudo concomitante de apoptose celular, pela técnica do TUNEL, e pela expressão da proteina Bcl-2, em placentas de gestantes portadoras de diabete ou de hiperglicemia diária (grupo IB) diferenciou estes grupos. Observou-se maior indice apoptótico e menor expressão de Bcl-2 em placentas do grupo IB, com valores intermediários aos encontrados em relação às placentas de gestantes normoglicêmicas (grupo IA) e portadoras de diabete (grupo IIB) ${ }^{23}$.

O uso de insulina elevou a expressão Bcl-2 e diminuiu o índice apoptótico. A razão TUNEL/ Bcl-2 teve valor próximo de 1 no grupo tratado com insulina e maior que 2 no grupo que recebeu apenas dieta para controlar a hiperglicemia materna. Em outras palavras, a insulinoterapia favoreceu a condição fisiológica de apoptose e proliferação celular nas placentas de gestantes portadoras de hiperglicemia ${ }^{23}$.

O estudo morfométrico das placentas evidenciou maior número de vilosidades e vasos vilositários que, apesar da menor área individual, garantiu maior superficie de troca materno-fetal. Estes resultados mostraram que as placentas do grupo IB de Rudge sofrem adaptações para manter adequada capacidade funcional, mas facilitam a passagem de glicose para o feto em desenvolvimento, explicando a macrossomia fetal. Nas placentas das gestantes portadoras de diabete gestacional ou clínico se observa esta adaptação vascular - apresentam a mesma área vilositária do grupo controle, menor área vascular e menor indice de capilarização, o que determina incapacidade funcional, explicando a restrição de crescimento e o risco de hipóxia intra-uterina ${ }^{24}$.

Razão TUNEL / Bcl-2

A vascularização intravilositária, ou seja, a extensão da superficie de trocas materno-fetal, guarda relação direta com o indice de pulsatilidade (PI) na dopplervelocimetria da artéria umbilical. Nas gestações complicadas por diabete ou hiperglicemia diária, o valor alterado de IP associado à hiperglicemia materna se relacionou a alterações morfométricas de vasos e vilosidades placentárias, destacando-se como indicador da condição de hipóxia intra-uterina ${ }^{25}$.

Estes resultados evidenciaram que os níveis glicêmicos maternos podem alterar as características morfométricas das vilosidades terminais e respectivos vasos vilositários. As alterações parecem ser proporcionais: niveis discretamente elevados de glicemia materna estimulam a proliferação vascular e a ramificação vilositária, possivelmente em resposta a menor grau de hipóxia, e garantem as trocas materno-fetais. Niveis glicêmicos mais acentuados, proporcionais à gravidade do quadro clínico materno e à hipóxia intrauterina, inibem a angiogênese vilositária, dificultam as trocas materno-fetais e aumentam o risco de hipóxia fetal e neonatal ${ }^{24}$.

Confirma-se a etiologia metabólica da resistência aumentada ao fluxo placentário e conseqüente prejuízo da oxigenação fetal, diretamente dependente da qualidade do controle metabólico materno. Do ponto de vista prático, o controle metabólico materno adequado e a presença de IP normal na artéria umbilical tranqüilizam o obstetra. Por outro lado, o resultado alterado de IP na artéria umbilical, associado a controle metabólico materno inadequado, deve ser valorizado e interpretado como sinal de insuficiência placentária ${ }^{25}$.

\section{Hiperglicemia materna e risco de desenvolvi- mento futuro de diabete}

Dang et al. ${ }^{26}$ observaram que o índice de massa corporal pré-gestacional era maior nas mães de recém-nascidos macrossômicos $(\mathrm{p}=0,008)$, sendo este índice a única variável indicativa de macrossomia. As gestantes do grupo IB de Rudge et al. ${ }^{9}$ são portadoras de hiperglicemia diária, necessitam de insulina para controle, têm predisposição para a obesidade e simulam modelo da síndrome metabólica na gestação. A associação destas características está associada a risco para a hipertensão arterial na gestação ${ }^{27}$ (RR=5,9; IC 95\%: 3,39-10,58).

O critério estabelecido por O'Sullivan e Mahan $^{28}$ para definir os valores de normalidade para o TTG100g na gravidez foi o risco de desenvolvimento futuro de diabete melito, e o que foi posteriormente confirmado por outros autores ${ }^{29-}$ ${ }^{31}$. Assim, o diabete melito gestacional, como definido e diagnosticado atualmente, caracteriza predisposição da mulher para desenvolver o diabete tipo 2 após o parto.

O seguimento destas mulheres, por um período de até 12 anos após a gravidez-índice, confirmou diabete clínico, tipo 2, em 16,7\% das gestantes classificadas no grupo IB de Rudge, em $23,6 \%$ das do grupo IIA e em 44,8\% naquelas do grupo IIB $^{32}$.

Em outras palavras, as gestantes portadoras 
de hiperglicemia diária (IB) e de diabete gestacional (IIA) apresentaram o mesmo risco para o desenvolvimento de diabete tipo 2 num periodo de até 12 anos. De acordo com Gabbe e Graves ${ }^{33}$, estas mulheres precisam ser identificadas, devem ser esclarecidas e têm direito a intervenções após a gestação para a prevenção do diabete melito no futuro.

\section{Conclusões e Perspectivas Futuras}

As gestantes do grupo IB apresentam rastreamento positivo e diagnóstico negativo de diabete gestacional, mas apresentam hiperglicemia diária, ou seja, alterações apenas do $\mathrm{PG}^{9}$. A resposta alterada ao TTG100g destas gestantes não é confirmada, provavelmente, porque os pontos de corte do TTG100g são elevados. Os melhores critérios para identificar recém-nascidos GIG/macrossômicos foram média para o TTG100g e média+um desvio-padrão $(\mathrm{m}+\mathrm{dp})$ para o $\mathrm{PG}^{22}$, valores já preconizados por Rudge ${ }^{9}$.

As gestantes do grupo IB correspondem a $13,8 \%$ da população com rastreamento positivo para diabete ${ }^{21}$, têm risco aumentado para hipertensão, obesidade ${ }^{31}$ e desenvolvem o mesmo percentual de diabete tipo 2 que as diabéticas gestacionais 10 a 12 anos após a gravidez-índice ${ }^{3}$.

Os recém-nascidos de mães portadoras de hiperglicemia diária têm a mesma incidência de macrossomia fetal que os filhos de mães portadora de diabete gestacional e as mesmas taxas de mortalidade perinatal, de hipoglicemia, de outros distúrbios metabólicos e respiratórios neonatais sindrome do desconforto respiratório e de malformações congênitas ${ }^{16,23}$. Em síntese, os recém-nascidos deste grupo específico de gestantes (IB) apresentam as mesmas repercussões do diabete materno, merecendo diagnóstico e tratamento precoces para a prevenção do resultado perinatal adverso. O risco atribuivel (RA) de morte perinatal neste grupo é de $4,16 \%$, comparável ao identificado nos grupos IIA + IIB $(6,12 \%)$.

As alterações placentárias do grupo IB são comparáveis às observadas em gestações complicadas pelo diabete materno, identificadas por características histopatológicas ${ }^{22,23}$, morfométricas e funcionais ${ }^{21,24-26}$.

Estas gestantes e seus recém-nascidos correspondem a uma população de alto risco na gravidez para o desenvolvimento futuro de diabete melito que não está sendo diagnosticada e tratada pelos profissionais de saúde. Seus recém-nascidos apresentam o mesmo potencial de compli- cações que os filhos de mães portadoras de diabete gestacional, incluindo o risco de morte perinatal. Assim, o grupo IB de Rudge, com taxa de morte perinatal de $41 \%$ o nascidos vivos, pode estar contribuindo de forma significativa para o aumento destes índices nas gestantes de baixo risco. A adoção do perfil glicêmico na assistência pré-natal das gestantes com triagem positiva, método de baixo custo e elevada acurácia para o diagnóstico dos distúrbios hiperglicêmicos de intensidade variada na gestação, é necessária pelo impacto na saúde da mulher e de seu recém-nascido.

Propõe-se o desenvolvimento de projeto temático, com participação de outros centros de pesquisa e de pesquisadores com múltiplos enfoques, incluindo financiamento por agências de fomento à pesquisa. Acreditando na necessidade de validar este grupo de gestantes para estabelecer protocolos diagnósticos e terapêuticos para a mãe, feto e recém-nascido, este projeto terá como objetivo a identificação de biomarcadores clínicos, metabólicos, bioquímicos, genéticos, imunológicos, celulares e moleculares, na forma de unidades mensuráveis, capazes de caracterizar a complicação obstétrica denominada aqui de hiperglicemia diária.

\section{Referências}

1. Cunningham FG, Leveno KJ, Bloom SL, Hauth JC, Gistrap III LC, Wenstrom KD. Williams obstetrics. 22nd ed. New York: McGraw-Hill; 2005.

2. HAPO Study Cooperative Research Group. The Hyperglycemia and Adverse Pregnancy Outcome (HAPO) Study. Int J Gynaecol Obstet. 2002;78(1):6977.

3. American Diabetes Association. Clinical practice recommendations. Diabetes Care. 2005;28 Suppl 1:S1-79.

4. Reichelt AJ, Oppermann MLR, Schmidt MI. Recomendações da $2^{a}$ reunião do grupo de trabalho em diabetes e gravidez. Arq Bras Endocrinol Metab. 2002;46(5):574-81.

5. Rudge MVC, De Luca LA. Diabetes e gravidez. Femina. 1981;9(6):463-7.

6. Rudge MVC, Calderon IMP, Ramos MD, Brasil MAM, Peraçoli JC. Comparação de dois métodos de rastreamento do diabete na gestação. Rev Bras Ginecol Obstet. 1994;16(6):203-5.

7. Ayach W, Calderon IMP, Rudge MVC, Costa RAA. Associação glicemia de jejum e fatores de risco como teste para rastreamento do diabete gestacional. Rev Bras Saúde Matern Infant. 2005;5(3):329-35. 
8. World Health Organization. Definition, diagnosis and classification of diabetes mellitus and its complications: report of a WHO consultation. Geneva: WHO; 1999.

9. Rudge MV, Peracoli JC, Berezowski AT, Calderon IM, Brasil MA. The oral glucose tolerance test is a poor predictor of hyperglycemia during pregnancy. Braz J Med Biol Res. 1990;23(11):1079-89.

10. Gillmer MD, Beard RW, Brooke FM, Oakley NM. Carbohydrate metabolism in pregnancy. Part I. Diurnal plasma glucose profile in normal and diabetic women. Br Med J. 1975;3(5980):399-402.

11. Pedersen J. A diabética grávida e seu recém-nascido. São Paulo: Manole; 1979.

12. Metzger BE. Biphasic effects of maternal metabolism on fetal growth: The quintessential expression of fuel-mediated teratogenesis. Diabetes. 1991;40 Suppl 2:99-105.

13. Sermer M, Naylor CD, Gare DJ, Kenshole AB, Ritchie JW, Farine D, et al. Impact of increasing carbohydrate intolerance on maternal-fetal outcomes in 3637 women without gestational diabetes. The Toronto Tri-Hospital Gestational Diabetes Project. Am J Obstet Gynecol. 1995;173(1):146-56.

14. Rudge MVC, Calderon IMP, Ramos MD, Maestá I, Souza LMS, Peraçoli JC. Perspectiva perinatal decorrente do rígido controle pré-natal em gestações complicadas pelo diabete. Rev Bras Ginecol Obstet. 1995; 17(1):26-32.

15. Rudge MV, Calderon IM, Ramos MD, Abbade JF, Rugolo LM. Perinatal outcome of pregnancies complicated by diabetes and by maternal daily hyperglycemia not related to diabetes. A retrospective 10-year analysis. Gynecol Obstet Invest. 2000;50(2):108-12.

16. Rudge MVC, Sgarbosa F, Dias CA, Calderon IMP, Costa C, Rugolo Junior A, et al. Aspectos obstétricos da macrossomia fetal. J Bras Ginecol. 1990;100(9):281-5.

17. Rudge MVC, Luca LA, Brasil MAM. Diagnóstico do diabete na gestação: I - Teste de tolerância oral á glicose. Rev Bras Ginecol Obstetr 1987; 4: 94-9.

18. Rudge MVC, Lima GR, Melo E, Luca LA. Diagnóstico do diabetes na gestação: II. Perfil glicêmico. Rev Bras Ginecol Obstet. 1988;10(1):47-50.

19. Backx CJ, Lotgering FK, Cornelis H, Wallenburg S. Oral glucose tolerance test is a poor predictor of hyperglycemia during pregnancy. J Perinat Med. 1989; 17(4):253-7.

20. Rudge MVC, Calderon IMP, Ramos MD, Suetake H, Peraçoli JC. Investigação diagnóstica do diabetes na gestação. Rev Bras Ginecol Obstet. 1996;18(1): 21-8.
21.Lima CP, Rudge MVC, Calderon IMP. Alterações histopatológicas das placentas de gestações complicadas pelo diabete materno. Femina. 1999;27(1):53-6.

22. Calderon IMP, Lima CP, Rudge MVC, Napoli G, Jeckel Neto EA, Ramos MD. Relação entre a classificação clínica de White e a histopatologia das placentas de gestantes diabéticas. Rev Bras Ginecol Obstet. 2000;22(7):401-11.

23. Sgarbosa F, Barbisan LF, Calderon IMP, Brasil MAM, Costa E, Gonçalves CR, Bevilacqua E, Rudge MVC. Changes in apoptosis and decreased Bcl-2 expression in human hyperglycemic, term placental trophoblast. Placenta. In press 2005.

24. Calderon IMP, Amorin RL, Damasceno DC, Costa RAA, Brasil MAM, Rudge MVC. Morphometric study of placental villi and vessels in women with diurnal hyperglycemia and gestational and clinical diabetes. Placenta. In press 2005.

25. Calderon IMP, Consonni M, Magalhães CG, Amorin RL, Brasil MAM, Rudge MVC. Doppler velocimetric study of umbilical artery and morphometric changes of placental villi and vessels in pregnant women with hyperglycaemia. BJOG. In press 2005.

26. Dang K, Homko C, Reece EA. Factors associated with fetal macrossomia in offspring of gestational diabetic women. J Matern Fetal Med. 2000;9(2):1147.

27. Pauletti TAVL, Rudge MVC, Calderon IMP, Peraçoli JC, Costa RAA. Síndrome de resistência à insulina: doença atual porém desconhecida. Femina. 2002;30(4):233-6.

28. O'Sullivan JB, Mahan CM. Criteria for the oral glucose tolerance test in pregnancy. Diabetes. 1964: 13:278-85.

29. Catalano PM, Vargo KM, Bernstein IM, Amini SB. Incidence and risk factors associated with abnormal postpartum glucose tolerance in women with gestational diabetes. Am J Obstet Gynecol. 1991;165(4 Pt1):914-9.

30.Kjos SL, Peters RK, Xiang A, Henry OA, Montoro $\mathrm{M}$, Buchanan TA. Predicting future diabetes in Latino woman with gestational diabetes. Utility of early postpartum glucose tolerance testing. Diabetes. 1995;44(5):586-91.

31. Hadden DR. A historical perspective on gestational diabetes. Diabetes Care. 1998;21 Suppl 2:B3-4.

32. Silva MG, Calderon IMP, Gonçalves LC, Aragon FF, Padovani CR, Pimenta WP. Ocorrência de diabetes melito em mulheres com hiperglicemia em gestação prévia. Rev Saúde Pública. 2003;37(3):345-50.

33. Gabbe SG, Graves CR. Management of diabetes mellitus complicating pregnancy. Obstet Gynecol. 2003;102(4):857-68. 